\title{
The CMS electromagnetic calorimeter
}

\author{
Francesca Cavallari* \\ INFN Romal, Italy \\ (on behalf of the CMS ECAL Collaboration) \\ E-mail: Francesca.Cavallari@romal.infn.it
}

From 2007 the new CERN proton proton collider will start collisions that could open new perspectives in the fields of high energy particle physics. The CMS experiment at the LHC is in advanced construction phase. In particular new physics could be discovered thanks to the CMS precise electromagnetic calorimeter (ECAL) made of about 75000 scintillating $\mathrm{PbWO}_{4}$ crystals. A description of the ECAL will be given and then the talk will focus on the recent progress in the detector and on the expected performance. In particular, based on the most recent test-beam results the talk will review the energy and position resolution, the monitoring system and the calibration methods, so crucial to maintain the design resolution. Finally the expected performance on few selected physics channels will be reviewed.

International Europhysics Conference on High Energy Physics

July 21st - 27th 2005

Lisboa, Portugal

${ }^{*}$ Speaker. 


\section{Introduction}

From 2007 the new CERN proton proton collider will start taking data at $\sqrt{s}=14 \mathrm{TeV}$. The CMS experiment [1] is one of the multi-purpose experiments built to explore the $\mathrm{TeV}$ energy range and search for new particles at the LHC. One of the most important searches that will be performed is the search for the Higgs boson $(\mathrm{H})$. The current exclusion limit was set by the LEP experiments at $114.4 \mathrm{GeV}$ [2]. The LHC may extend this search up to $1 \mathrm{TeV}$, the theoretical highest mass bound for this particle, thus covering the full search range. In addition precision fits to electro-weak measurements indicate that the preferred region for the $\mathrm{H}$ mass is rather close to the exclusion limit [3]. In this range the golden channel in the search is via the $\mathrm{H} \rightarrow \gamma \gamma$ decay. The cross-section times B.R. for this process is small, while the background is constituted by events with jets with some real or some mis-identified $\gamma$ and it is large. Thus a mass resolution better than $1 \%$ for the two $\gamma$ is required so that the $\mathrm{H}$ mass peak can be identified over the continuous mass background. This requires a very good energy and position resolution for high energy $\gamma$. To meet these requirements the CMS experiment has decided to build a homogeneous electromagnetic calorimeter.

\section{The CMS Electromagnetic Calorimeter}

The CMS electromagnetic calorimeter (ECAL) is made of about 75000 lead tungstate $\left(\mathrm{PbWO}_{4}\right)$ scintillating crystals [4] . It is subdivided in a central barrel part (between $\eta=0$ and $\eta=1.48$ ) and two endcaps (up to $\eta=3$ ). A silicon and lead pre-shower will be installed in front of the endcaps to improve the position resolution there.

The energy resolution of an electromagnetic calorimeter can be parametrized as:

$$
\frac{\sigma(E)}{E}=\frac{a}{\sqrt{E}} \bigoplus \frac{b}{E} \bigoplus c
$$

where $a$ is the stochastic term (due to the statistical fluctuations in the shower and in the detector response), $b$ contains the electronic noise contribution and $c$ is the so-called constant term which takes care of possible changes in channel calibration and system instabilities, as well as energy leakage or dead materials. For a crystal calorimeter the stochastic term is small and, with adequate electronics, the noise term is also small, so the dominating contribution at high energy is the constant term $c$. Special care has been devoted in the ECAL to have this term at the level of $0.55 \%$.

The LHC is a demanding environment for the detectors: due to the high bunch crossing rate (of $25 \mathrm{~ns}$ ) detectors must be fast; radiation hardness is an issue due to the high particle fluences in the detectors; high granularity and compactness are required for a collider experiment; and the solenoid provides a $4 \mathrm{~T}$ magnetic field in the central part of the experiment, so magnetic field tolerant devices are required.

The choice of the $\mathrm{PbWO}_{4}$ as a scintillating crystal for the CMS ECAL was driven by these requirements: the scintillation light is emitted fast (more than $80 \%$ of the light can be collected in $25 \mathrm{~ns}$ ), it is radiation tolerant up to the LHC dose, it is very dense, so the radiation length is small $\left(\mathrm{X}_{0}=0.89 \mathrm{~cm}\right)$ and the Moliere radius is $2.2 \mathrm{~cm}$ so the detector could be made compact. However its light yield (LY) is low. 
For what concerns the photon sensors, Avalanche Photodiodes (APD) have been chosen in the Barrel and Vacuum Photo-triodes in the endcaps. APDs are silicon photodiodes with internal amplification, they are compact, radiation hard up to the LHC neutron fluence in the barrel, magnetic field insensitive and they are typically operated at gain 50. Two APDs are coupled to each crystal, with a total active area of $0.5 \mathrm{~cm}^{2}$. All ECAL APDs are already produced and tested. VPTs are single gain stage photomultiplier tubes, they have a gain of typically 10 when there is no magnetic field and a gain reduction of $15 \%$ at $4 \mathrm{~T}$. Almost all of the ECAL VPTs are produced and tested.

The ECAL crystals are $23 \mathrm{~cm}$ in length and constitute more than $25 X_{0}$, thus the contribution to the stochastic term given by containment is small $(a=1.5 \% / \sqrt{E})$. The $\mathrm{LY}$ of the $\mathrm{PbWO}_{4}$ coupled to both types of photon sensors gives a $\mathrm{LY}=4$ p.e./MeV, thus adding a small contribution to the stochastic term of $a=2.3 \% / \sqrt{E}$.

CMS $\mathrm{PbWO}_{4}$ crystals are mostly being produced by Bogoroditsk BTCP plant in Russia. As of today, more than $50 \%$ of the crystals are already produced and mechanically assembled in modules. In addition, to have more contingency, the ECAL collaboration has decided to buy 2700 barrel crystals and 2950 endcap crystals from the Shangai Institute of Ceramics (SIC, China). According to the current schedule, the ECAL barrel will be ready for data taking in 2007 and the endcaps and pre-shower will be installed after the first shutdown.

All the relevant crystal characteristics are controlled by automatic machines (ACCOS [5]) in the two regional centres for the calorimeter assembly (at CERN and in Rome at INFN-ENEA center). Among these properties some have been shown to potentially influence the constant term $c$ of the calorimeter energy resolution. In particular the position of the electromagnetic shower maximum may fluctuate due to the fluctuations in the depth of the first interaction of the impinging $e$ or $\gamma$. So the crystal LY must be uniform along the crystal depth, otherwise these fluctuations may spoil the energy resolution. The ECAL crystals have a tronco-piramidal shape that causes a geometrical focusing of the light from the front face towards the rear face. Thus the crystals are naturally non uniform. In order to compensate for this effect, one of the crystal lateral faces is depolished at the factory. This causes a mild loss of light but improves the LY uniformity.

At the LHC the total dose of $\gamma$ in the barrel has been estimated to $2-4 \cdot 10^{3}$ Gy and the neutron fluence to $2 \cdot 10^{13} \mathrm{n} / \mathrm{cm}^{2}$. After a long $\mathrm{R} \& \mathrm{D}$ the $\mathrm{PbWO}_{4}$ crystals are now radiation tolerant [6]. The scintillation mechanism is not affected but the $\gamma$ radiation causes a transparency loss that saturates quite fast. The damage recovers in few hours without radiation. The crystal radiation resistance is well correlated with its original optical transmission so it can be estimated with a simple optical measurement. The crystals whose transmission curve indicates a poor radiation resistance undergo a test irradiation.

In the LHC environment it is mandatory to monitor the crystal transparency changes due to irradiation. Thus a sophisticated monitoring system has been conceived for the CMS ECAL. Each crystal is illuminated via an optical fiber with laser light at $420 \mathrm{~nm}$, at the $\mathrm{PbWO}_{4}$ emission peak. A system of PN diodes monitors the amplitude of the laser pulses transmitted for each bunch of fibres.

The APD gain depends on the high voltage at the level of $3 \% /$ Volt at gain 50. The High Voltage system has been designed and qualified to mantain the required voltage over about 30 days with a maximum deviation of $66 \mathrm{mV}$. This guarantees that the maximum effect on the ECAL energy resolution constant term due to the $\mathrm{HV}$ is below $0.2 \%$. 
The crystal LY depends on the temperature at the level of $-2 \% / \mathrm{C}$, and the APD gain has a similar dependence, resulting in a total dependence of the channel calibration on the temperature of $-4 \% / \mathrm{C}$. Thus a very stable cooling system has been put in place to stabilize both the crystals and the photon sensors to better than $0.1 \mathrm{C}$.

The 2004 test-beam campaign with the first final module of 1700 crystals, which is the building block of the ECAL barrel, has shown that a stability of the temperature to better than $0.1 \mathrm{C}$ over more than a month of operation has been achieved and that the response of the monitoring system was stable at the level of $0.1 \%$. The average electronic noise in one channel is equivalent to $39 \mathrm{MeV}$ and there is no correlated noise. The stochastic term of the energy resolution is below $3 \% / \sqrt{E}$. The energy resolution measured for various crystals corresponding to various regions in $\eta$ confirms the achievement of a constant term of $0.4-0.45 \%$. So the additional contribution due to the channel inter-calibration in CMS must be of the same level in order to give $c=0.55 \%$.

\section{The ECAL calibration prior to LHC start-up}

The ECAL calibration prior to LHC start-up is fundamental both for the detector operation and for the early discovery potential for new physics. As of construction, the calorimeter channels will be different and mis-calibrated at the level of 6-10\%, due to intrinsic differences among individual $\mathrm{PbWO}_{4}$ crystals, quantum efficiency of the photo-detectors and response of the read-out electronics.

At the start-up three methods are envisaged to inter-calibrate the channels:

- a preliminary inter-calibration can be deduced from the crystals and APDs laboratory measurements and it is proved [7] to give a resolution of about 4\%,

- the exposure to cosmics rays for one-two weeks of one module in 2004 has given a resolution of about $2-3 \%$,

- if possible some modules will be calibrated with high energy electron beams to reach a calibration at $0.5 \%$ level.

\section{In-situ calibration}

In CMS the ECAL can be calibrated with physics events. At the beginning of the detector operation a fast inter-calibration method can be used, based on the $\Phi$ symmetry in jet events [8]. This method is based on the principle that the average energy deposited over a ring of crystals in $\Phi$ must be equal. After a selection of events with at least one energetic jet, the region around the triggering jet is escluded, and then all the hits between 1 and $6 \mathrm{GeV}$ are used to calculate the average energy per channel. A Monte Carlo simulation has shown that the limit of this method is the non perfect $\Phi$ symmetry of the detector and that a calibration at the level of about 1-2\% can be obtained in the barrel and between 1-3\% in the endcaps.

The $\Phi$ rings can be rapidly inter-calibrated using $Z \rightarrow e^{+} e^{-}$events by reconstruction of the invariant mass. The $Z \rightarrow e^{+} e^{-}$rate is about $1 \mathrm{~Hz}$ at low luminosity and 100 electrons per ring are enough to inter-calibrate at the $0.5 \%$ level. Such statistics can be obtained in less than a day. 
When the Tracker is fully functional also $W \rightarrow e v_{e}$ events can be used to calibrate the ECAL. This method minimizes the difference between the momentum of the electron track measured in the Tracker and the energy in the ECAL. A Monte Carlo simulation of this process has shown that the main difficulty is the Bremsstrahlung in the Tracker material in front of the ECAL. So the event selection is aimed at rejecting events with high Bremsstrahlung. The calibration precision attained depends on $\eta$ and for an integrated luminosity of $5 \mathrm{pb}^{-1}$ it may reach $0.5-1.0 \%$ resolution in the barrel, the method limit being below $0.5 \%$ with higher statistics.

\section{First physics with the CMS ECAL}

At the LHC start-up the ECAL will be commissioned with physics events using well known signals like the $Z \rightarrow e^{+} e^{-}$events. But there are few kinds of signals of new physics that the ECAL can immediately see, even with little integrated luminosity, like the existence of high mass $e^{+} e^{-}$ resonances. Such resonances are predicted in some models as for example the Randall-Sundrum model with 1 extra dimension predicts the possibility of observing at the LHC one high mass excitation of the graviton that may decay to $e^{+} e^{-}$. The early accessibility of such channel depends on the coupling constant $c_{R S}$ in the model and on the graviton resonance mass. A Monte Carlo simulation [10] has shown that even in the pessimistic case of $c_{R S}=0.01$, an integrated luminosity of $1.2 \mathrm{fb}^{-1}$ would be enough to explore the mass range up to $900 \mathrm{GeV}$ while $8 \mathrm{fb}^{-1}$ are required for a mass of $1.25 \mathrm{TeV}$.

\section{References}

[1] CMS, The Compact Muon Solenoid, Technical Proposal, CERN/LHCC 94-38.

[2] see presentations in the session Physics beyond the SM in these proceedings.

[3] M. Grünewald, SM precision tests, in the section Tests of SM of these Proceedings.

[4] CMS, the Electromagnetic Calorimeter Project, Technical Design Report, CERN/LHCC 97-33.

[5] E. Auffray, et al., CMS NOTE 1999/067; S. Baccaro et al., NIM A 459 (2001) 278-284.

[6] A. Annenkov et al., CMS NOTE 1997/008,

S. Baccaro et al, CMS NOTE 2000/002.

[7] F. Cavallari et al., CMS RN 2004/002.

[8] D. Futyan, CMS Note 2004/007.

[9] L.Randall and R.Sundrum, Phys. Rev. Lett. 83 (1999) 3370, and Phys. Rev. Lett. 83 (1999) 4690.

[10] C.Collard, M-C.Lemaire, CMS Note 2004/024, Eur. Phys. J. C40 (2005), Num 5, 15.

CMS Documents are available at http:// cmsdoc. cern. ch/cms.html. 